\title{
A family of metric gravities
}

\section{With a new exact Schwarzschild method, a local space-time relation, strong-field verification targets, and the ability to promote field gravities to full metricity}

\author{
Robert Shuler ${ }^{\mathrm{a}}$ \\ NASA Johnson Space Center, 2101 NASA Parkway/EV2, Houston, TX 77058, USA
}

Received: 26 July 2017 / Revised: 16 February 2018

Published online: 23 April 2018

(C) The Author(s) 2018. This article is published with open access at Springerlink.com

\begin{abstract}
The goal of this paper is to take a completely fresh approach to metric gravity, in which the metric principle is strictly adhered to but its properties in local space-time are derived from conservation principles, not inferred from a global field equation. The global field strength variation then gains some flexibility, but only in the regime of very strong fields (2nd-order terms) whose measurement is now being contemplated. So doing provides a family of similar gravities, differing only in strong fields, which could be developed into meaningful verification targets for strong fields after the manner in which far-field variations were used in the 20th century. General Relativity (GR) is shown to be a member of the family and this is demonstrated by deriving the Schwarzschild metric exactly from a suitable field strength assumption. The method of doing so is interesting in itself because it involves only one differential equation rather than the usual four. Exact static symmetric field solutions are also given for one pedagogical alternative based on potential, and one theoretical alternative based on inertia, and the prospects of experimentally differentiating these are analyzed. Whether the method overturns the conventional wisdom that GR is the only metric theory of gravity and that alternatives must introduce additional interactions and fields is somewhat semantical, depending on whether one views the field strength assumption as a field and whether the assumption that produces GR is considered unique in some way. It is of course possible to have other fields, and the local space-time principle can be applied to field gravities which usually are weak-field approximations having only time dilation, giving them the spatial factor and promoting them to full metric theories. Though usually pedagogical, some of them are interesting from a quantum gravity perspective. Cases are noted where mass measurement errors, or distributions of dark matter, can cause one theory to mimic another implying that such estimates or distributions should be first obtained from weakfield measurements before being used to discriminate verification candidates. By this method theorists gain insight into the local constraints on space-time, and GR verification gains strong-field comparative objectives.
\end{abstract}

\section{Introduction}

In typical developments of General Relativity (GR) [1-7] Riemannian geometry and the tensor calculus are assumed. Then a field equation is postulated heuristically, often though not exclusively starting from Poisson's equation and analogy with Newtonian gravity [2-5] but sometimes with unique rationale such as conservation of the source curvature [6]. Often the field equation is justified partly with the observation that it is the simplest possible or even only one, as for example: "Under certain assumptions about the structure of physical theories, and of the properties of the gravitational field, General Relativity is the only theory that describes gravity. Alternative theories introduce additional interactions and fields [8]." This claim originated with Einstein and continues in the modern texts cited. Mass-energy is coupled only to the metric, and variations, either serious theories or verification models, are developed using fields which influence the metric. Since alternatives are essentially ad hoc modifications, the process of developing and evaluating them usually requires an exhaustive consideration of compatibility with existing data, conservation principles and successful predictions of the existing well-verified theory. This substantial difficulty now borne separately by each proposed alternative. While it served well to produce verification targets in the 20th century for astrophysical and

\footnotetext{
a e-mail: robert@mc1soft.com
} 
cosmological distances, at a time when GR was less well verified, there are fewer viable theoretical alternatives for strong fields. Strong fields could see verification activity in the 21 st century. The approach of this paper guarantees all members of a "family" of theories inherit the same common behavior in weak fields. One needs only scrutinize their strong-field behavior.

While in some sense we intend a simpler or partial method, we do not want a method based on simplifying assumptions which are not theoretically valid [9]. This paper presents a "quick-look" or "minimalist" framework based on a consistency of field strength measurement postulate, implicitly contained in GR and originally developed to evaluate the relation between inertia and gravity [10]. The framework will be re-developed more formally without excess reliance on symmetry, extended to dynamic fields, and three examples worked out including the exact Schwarzschild solution in the GR case. The term "minimalist" refers to a minimal basis of assumptions and results common to all family members.

One key to rapid formulation is to forego the complete generality of tensors and to use a frame of reference which permits conservation laws. We take a frame of reference to be coordinates, in most cases asymptotic to the space-time manifold at some point, in which quantities may be meaningfully compared, as opposed to arbitrary coordinates. We know from Noether's theorem these are associated with homogeneous spaces [11], with a uniform time required for conservation of energy, and uniform spatial intervals required for conservation of momentum. It might seem immaterial since the gravitational field is active in modifying momentum and energy, but our heuristic for this paper will be to choose homogeneous observer coordinates while otherwise following the general pattern of metric gravity development. We will thus be able to make postulates in terms of conservation principles and minimize the confusion of non-intuitive coordinate systems. For topologies not readily mapped to a homogeneous space there would seem to be two options, both of which are useful, and they are not mutually exclusive:

1) One can presume coordinate singularities are no more meaningful than in the standard theory. Conservation of momentum and energy across a singularity in an otherwise conservative homogeneous space is without definition, since no real physical measurement can be made in finite time.

2) In some cases we can adopt a non-flat but still homogeneous frame of reference. This would work for many cosmological solutions.

A second goal of this paper will be to see if additional insight emerges, and it appears so.

To begin, we adopt a basis for a metric using coordinates where $r=C / 2 \pi$, which is homogeneous in $r$. Since this metric will have no off-diagonal elements its standard form is

$$
\mathrm{d} s^{2}=-\mathrm{d} \tau^{2} c^{2}=g_{t t} \mathrm{~d} t^{2}+g_{r r} \mathrm{~d} r^{2}+g_{\theta \theta} \mathrm{d} \theta^{2}+g_{\phi \phi} \mathrm{d} \phi^{2}
$$

We take (1) and geodesic motion as our geometric gravity postulate. Restricting ourselves to the neighborhood of a particular point on radial axis $r$ we adopt Cartesian observer coordinates $(\mathrm{d} t, \mathrm{~d} r, \mathrm{~d} y, \mathrm{~d} z)$ that are locally homogeneous in all dimensions by assuming $g_{\theta \theta}=r^{2}, g_{\phi \phi}=r^{2} \sin ^{2} \theta$ which are just spherical-to-Cartesian transforms. The problem for the static symmetric case is to find functions $g_{t t}(r, M)$ and $g_{r r}(r, M)$ where $M$ is the source mass, using whatever postulates we need to replace the heuristic justification of the field equation in GR, remain consistent with the principle of relativity, and match Newtonian gravity as $r \rightarrow \infty$. Once suitable postulates are identified and confirmed, the spherical symmetry will be loosened and the relation to dynamic and cosmological solutions discussed.

We then re-solve the static field problem, investigating postulates that must be made in lieu of the field equation and whether any relation between $g_{t t}$ and $g_{r r}$ is a coordinate artifact or something of physical interest. This should provide a model for rapid investigation of other postulates and fields, which we demonstrate with two non-GR examples. In this way we provide verification targets which are not just based on arbitrary parameters, but on physically meaningful alternatives, and also provide a framework to aid in developing and evaluating such alternatives.

\section{Methods}

\subsection{Coordinate quantities and frames of reference}

Local coordinates at any point $\mathbf{x}_{R}$ in the field will be $(\mathrm{d} \tau, \mathrm{d} l, \mathrm{~d} y=r \mathrm{~d} \theta, \mathrm{d} z=r \sin \mathrm{d} \phi)$ where $\mathrm{d} l$ is a local spatial displacement $\mathrm{d} s$ aligned with the field acceleration $g$ and radial axis $r$. This frame of reference which we call $\mathbf{R}_{\mathrm{R}}$ is local to a point at radius $r$. Measurements made near point $\mathbf{x}_{R}$ will be subscripted " $R$ " if vector and " $r$ " if scalar, for example force $\mathbf{F}_{R}$, velocity $\mathbf{v}_{R}$, acceleration $\mathbf{a}_{R}$, and gravitational acceleration $g_{r}$. Observer coordinates we take to be with reference to an observer at $r \rightarrow \infty$ or at least such distance that time dilation is negligible. The point $\mathbf{x}_{R}$ expressed in observer coordinates is $\mathbf{x}_{c}$ associated with $(\mathrm{d} r, \mathrm{~d} r, \mathrm{~d} y=r \mathrm{~d} \theta, \mathrm{d} z=r \sin \theta \mathrm{d} \phi)$. This frame of reference we call $\mathbf{R}_{\mathrm{C}}$ for coordinate observer reference. 
A "c" indicates an "observer coordinate" quantity, but usually we will not write the "c" assuming that unsubscripted $\mathbf{x}$ is always $\mathbf{x}_{c}$. Two cases are not clear unless we write the subscript. The coordinate velocity of light will be written $\mathbf{c}_{c}$. The second case will be noted later.

Our analysis will avoid relativistic speeds so that it is easy to work with coordinate space-time ratios $\boldsymbol{\rho}_{S T}$ defined as follows:

$$
\boldsymbol{\rho}_{S T}=\left(\rho_{t t}, \boldsymbol{\rho}_{R}\right)=\left(\rho_{t t}=\frac{\mathrm{d} \tau}{\mathrm{d} t}, \rho_{r r}=\frac{\mathrm{d} l}{\mathrm{~d} r}, \rho_{y y}=\frac{\mathrm{d} y_{r}}{\mathrm{~d} y}, \rho_{z z}=\frac{\mathrm{d} z_{r}}{\mathrm{~d} z}\right)=\left(\frac{\sqrt{-g_{t t}}}{c}, \sqrt{g_{r r}}, 1,1\right)
$$

For spherical symmetry we expect $\rho_{y y}=\rho_{z z}=1$ as shown in (2), though not for the general case. Additionally, we use $\varsigma=\rho_{t t}^{-1}$ for the "time dilation factor" when we look for the sense of magnitude of potential energy and thus field strength. One can think of $\varsigma$ as representing field strength.

We have at low velocities the following coordinate transformations for locally measured quantities in $\mathbf{R}_{\mathrm{R}}$ expressed in the observer reference $\mathbf{R}_{\mathrm{C}}$, which we take to be at $r_{\text {observer }} \rightarrow \infty$ or at least such distance that time dilation is negligible (e.g., $v_{r}$ means local at $r$, not radial velocity, and coordinate velocities at $r_{\text {observer }}$ are on the left):

$$
\begin{aligned}
\mathrm{d} t & =\varsigma \mathrm{d} \tau, \\
\mathrm{d} l & =\rho_{r r} \mathrm{~d} r \Leftrightarrow \mathrm{d} r=\mathrm{d} l / \rho_{r r}, \\
v_{\text {transverse }} & =v_{r \text {-transverse }} / \varsigma, \\
a_{\text {transverse }} & =a_{r \text {-transverse }} / \varsigma^{2}, \\
v_{\text {radial }} & =v_{r \text {-radial }} \rho_{r r} / \varsigma, \\
a_{\text {radial }} & =a_{r \text {-radial }} \rho_{r r} / \varsigma^{2}, \\
g & =g_{r} \rho_{\text {rr }} / \varsigma^{2}, \\
c_{c \text {-transverse }} & =c_{\text {transverse }} / \varsigma, \\
c_{c-\text { radial }} & =c_{\text {radial }} \rho_{r r} / \varsigma .
\end{aligned}
$$

For a spherically symmetric solution definitions (3) are sufficient. We here introduce a more general vector form mainly to show how it is possible, using " $\circ$ " as the entrywise or Hadamard product (products of corresponding elements form a vector or matrix of the same dimension), $x^{\circ 2}$ as $x \circ x$, and "/" as the entrywise or scalar operation as applicable, and using $\mathrm{d} \mathbf{x}$ as a spatial vector:

$$
\begin{aligned}
\mathrm{d} \mathbf{x}_{R} & =\mathrm{d} \mathbf{x} \circ \boldsymbol{\rho}_{R} \Leftrightarrow \mathrm{d} \mathbf{x}=\mathrm{d} \mathbf{x}_{R} / \boldsymbol{\rho}_{R}, \\
\mathbf{v} & =\mathbf{v}_{r} \circ \boldsymbol{\rho}_{R} / \varsigma, \\
\mathbf{c}_{c} & =\mathbf{c} \circ \boldsymbol{\rho}_{R} / \varsigma, \\
\mathbf{a} & =\mathbf{a}_{r} \circ \boldsymbol{\rho}_{R} / \varsigma^{2} .
\end{aligned}
$$

\subsection{Conservation of proper momentum (CPM) postulate}

To get the transformation of a force transferred between two points, and two frames of reference, we need to make a postulate. This postulate will relate locally measured momentum (near the reference frame origin) in the two frames of reference $\mathbf{R}_{R}$ and $\mathbf{R}_{\mathrm{C}}$. The momentum will be transferred from one location to another by a tether or rod or lever according to what action is needed (pull or push, radial or transverse). No mass falls through the field, and local proper units are the same at each end, so we postulate that this momentum as locally measured is conserved.

Let a force be a series of momentum impulses. Note that $F=\mathrm{d}(m v) / \mathrm{d} t$ works as well for a time series of discrete $m v$ impulses as for continuous force. The effect of this assumption is that there are more momentum impulses per unit time on the bottom end of the rod-tether-lever by the amount of the time dilation factor, so that

$$
\begin{aligned}
\mathbf{F}_{r} & =m \mathbf{a}_{r}=\varsigma \mathrm{F} \\
F & =m \mathbf{a}_{r} / \varsigma=m \mathbf{a} \varsigma^{2} / \varsigma \boldsymbol{\rho}_{R}=m \mathbf{a} \varsigma / \boldsymbol{\rho}_{R} .
\end{aligned}
$$

A thought experiment for transferring momentum is shown in fig. 1. Any tethers or levers used are compliant as required by relativity, and may flex or may delay the momentum, but are assumed to accomplish lossless transfer of a proper length or a conserved momentum impulse (for the lever depending on fulcrum placement).

In other words, objects on a tether feel heavier at the bottom than the top because of the metric temporal factor. We assume that rest mass in the object (proper) frame is constant $m_{r}=m$ and define a new quantity, coordinate 


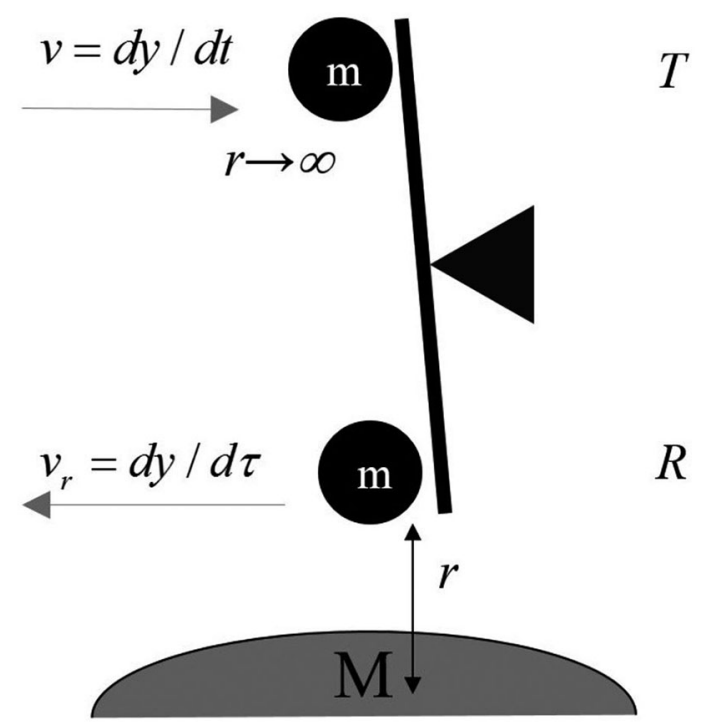

Fig. 1. Transfer of momentum in a gravitational field.

inertia $m_{c}$ (the second case in which "c" must be written for clarity), to represent the apparent inertia the distant observer feels when tugging on an object at $r$ and comparing force to coordinate distances in the frame $\mathbf{R}_{\mathrm{C}}$ :

$$
\mathbf{F}=m_{c} \mathbf{a}=\left(m \varsigma / \boldsymbol{\rho}_{R}\right) \mathbf{a}=m \mathbf{a}_{r} / \varsigma .
$$

The reader is cautioned against comparing the force felt at the top in $\mathbf{R}_{\mathrm{C}}$ with the length of fulcrum or tether displacement, as the latter is a proper length referred from $\mathbf{R}_{R}$. Measurement of coordinate lengths from $\mathbf{R}_{\mathrm{R}}$ in the frame $\mathbf{R}_{\mathrm{C}}$ can only be inferred from coordinate definitions, in this case from $r=C / 2 \pi$.

Equation (6) is isotropic with respect to local acceleration at $r$ but anisotropic with respect to coordinate acceleration because that depends on the spatial factor $\boldsymbol{\rho}_{R}$, and so is consistent both with experimental evidence for the isotropy of inertia in atomic oscillators and yet a kind of anisotropy as well [12]. However, if we connect the top and bottom with a tether which by definition spools out the same proper length on each end, then we can define tether lengths, velocity, acceleration and force at the top (observer coordinate frame $r \rightarrow \infty$, implicit when no subscript) which are independent of $\rho_{R}$ and therefore isotropic (and in the radial case are different than coordinate quantities) by omission of the $\boldsymbol{\rho}_{R}$ factor. We then have the tether relations in (7). Scalars are shown, because direction is arbitrary depending on the tether. The subscript tether indicates actual measurement at the top based on proper tether lengths, as opposed to a coordinate quantity. However, due to the conservation of proper momentum postulate $F_{\text {tether }}=F$.

$$
\begin{aligned}
\mathrm{d} l_{\text {tether }} & =\mathrm{d} l_{r} \\
v_{\text {tether }} & =v_{r} / \varsigma \\
a_{\text {tether }} & =a_{r} / \varsigma^{2} \\
F_{\text {tether }} & =F_{r} \varsigma .
\end{aligned}
$$

\subsection{Consistency of field strength measurement (CFS) postulate}

Suppose we measure the strength of the field at $r$ using a massless tether attached to a point mass $m$ and letting it spool out freely (we have a large spool of inertia-less tether and it is withdrawn without resistance), noting the acceleration at the top is $g_{\text {tether }}=g_{r} / \varsigma^{2}$ from the acceleration relation in $(7)$.

Now we make a second experiment in which a force is applied to the tether to exactly counter any acceleration. The tether force according to (8) is also a measure of field strength:

$$
F=m g \varsigma / \rho_{R}=m g \varsigma / \rho_{r r}=m g / \rho_{t t} \rho_{r r} .
$$


By postulate, we require these two measures to be equivalent, or to vary in proportion to each other $F \propto g$. For example, if one is constant over an interval the other is also, and so forth. One may think of this in terms of equivalence. Suppose a mass is dragged on a tether behind a rocket in constant acceleration $g$. If the tether has no mass itself, this postulate asserts that the inertia felt by the rocket is the same no matter the tether length. So it is physically reasonable. Since $m$ is a constant, and we have supposed $g$ constant, the only way that is going to happen is if

$$
\rho_{r r} \propto \rho_{t t}^{-1} \Leftrightarrow k_{r \varsigma}=\rho_{t t} \rho_{r r} \Leftrightarrow \rho_{r r}=k_{r \varsigma} \varsigma
$$

For a century (9) has been thought to be an artifact of coordinate choice. That the force on a tether is independent of the length of a massless tether can be measured locally, and is not dependent on coordinate choice. Working backward from Schwarzschild-GR, the force relation (6) can be found which is sufficient to imply that the CFS postulate holds in static symmetric GR. It is a physical principle, not a coordinate artifact.

However, it is a property of the local structure of space-time, not the global variation of field strength. Therefore it is still correct to say that GR cannot be deduced from equivalence alone, even with the consistency of field strength measurement (CFS). The significance of our approach is that by not postulating a field equation all at once, we can draw some separate conclusions about the local structure of space-time, and propose a family of theories (a framework) which includes GR and arbitrarily nearby theories. The CFS postulate can also be separately an object of empirical verification, upon which the entire family stands or falls.

\subsection{CFS postulate in dynamic and spatially varying fields}

We cannot immediately claim the CFS postulate over large distances in dynamic situations because the length of the tether affects the timing of the reflected tension transmitting the acceleration impulse and the return inertia retarding impulse. Also the trailing mass might literally bounce forward if the tether is stiff and the rocket makes a sudden acceleration impulse, providing up to a double inertial retarding impulse. But there might not be any response to the next acceleration impulse (depending on magnitude) due to tether slack. So we will claim that if there is a stable average value of acceleration, then the stable average value of the inertia of the mass felt at the rocket will not depend on tether length and the CFS postulate will then hold.

Further, if the stress propagation time along the tether is small compared to the rate of change of acceleration, then we can claim the CFS postulate approximately by noting the situation is quasi-static. In the limit as the tether length approaches zero, the CFS postulate is exactly true even in dynamic situations.

The rocket-and-mass equivalence analysis has another limitation that we will remove in like manner. It models only constant field strength. In the limit as tether length becomes arbitrarily small in comparison to distances over which field strength varies, the CFS postulate becomes true for spatially varying fields. The two together indicate that CFS is a property of local space-time for non-singular analytic field functions. In retrospect, it seems that these same limitations would apply to any Equivalence Principle basis for the formulation of gravity. It appears our method suffers no particular theoretical disadvantage with respect to the normal derivation of GR.

\subsection{Locally conserved space-time}

If we confine ourselves to a sufficiently small differential volume such that there is an approximately uniform acceleration, the effect on the spatial ratios $\mathbf{p}_{R}=\left(\rho_{r r}, \rho_{y y} \rho_{z z}\right)$ accumulates along one direction at a time, the direction of the gravitational acceleration $\hat{g}$. Define a product of all space and time ratios in space free of gravitational fields at $r \rightarrow \infty$ as

$$
k_{S T}=k_{r \varsigma} \rho_{x x} \rho_{y y}=\rho_{t t} \rho_{r r} \rho_{x x} \rho_{y y} .
$$

The constant $k_{S T}$ is a ratio of differential space-time volumes. If temporal units are longer, then length units must be shorter and vice versa. We have no way of knowing the spatial units' absolute value, or the ratio between any three orthogonal directions of $\boldsymbol{\rho}_{R}$ at a point in space even if free of gravitational fields, for the same reason that a free falling local observer in a gravitational field, confined to his local space, cannot tell that it is deformed. We can only measure coordinate ratios between points in space, and even that only indirectly such as by light bending, since we inherently cannot compare a measuring rod in one place or orientation to a measuring rod in another without moving or turning it.

This result appears as an intermediate step in some derivations of the Schwarzschild solution, but because the coordinates there are arbitrary it is not clear that it is a conservation principle. However, it seems clear from our derivation. 


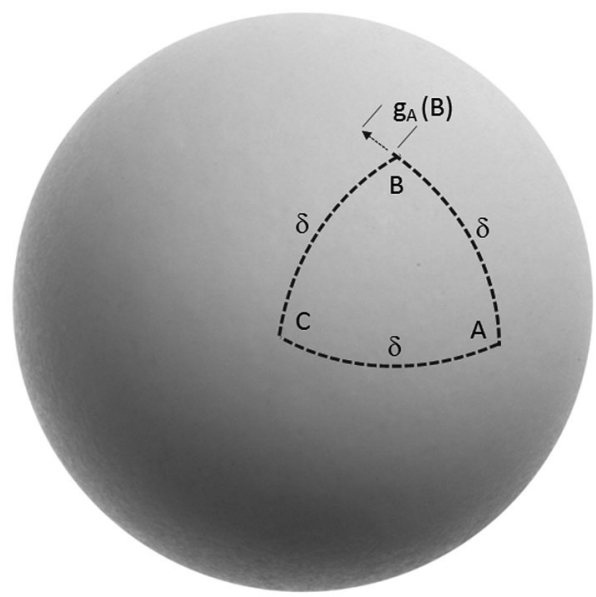

Fig. 2. Applying consistency of field strength measurement in expanding space-time.

\subsection{Time-varying and cosmological fields}

It is possible to imagine a time-varying field with approximately no acceleration component, as for example a small region between two approaching or receding masses and transverse to the axis between them. Our analysis shows no associated spatial distortions if there is no acceleration since in (5) we have $\mathbf{a}=0$.

For a symmetric cosmological expansion or contraction there is no variation in the spatial field for local masses. For a pair of objects at sufficient distance that they are not gravitationally bound, we expect to find a component of acceleration due to the expansion or contraction. But from the point of view of either of them, the local acceleration is still zero. Consider $\mathrm{A}, \mathrm{B}$ and $\mathrm{C}$ in fig. 2 separated by a common proper distance $\delta$ at a point in the time frame of co-moving observers. It is usual to characterize the expansion as a mutual recession velocity of all three along the lines between them. But as $\delta$ increases the recession velocity also increases since it is a function of $\delta$ by virtue of the Hubble constant. So there is a small acceleration of B with respect to A: $g_{A}(B)$, and so forth for each pair of points, and from each point of view.

We can imagine a series of thought experiments in which from each point we let out an ideal tether and measure the "field strength" associated with the acceleration at each other point. Comparing a freely spooling tether with slightly increasing velocity indicated by $g_{A}(B)$ etc. with the force on a tether restrictively spooled at a constant velocity (no acceleration, but a constant velocity of the initial recession velocity as it is the goal only to measure force associated with the acceleration), we can apply consistency of field strength measurement (9) and determine that locally about B in A's coordinates, in proportion to the increasing time dilation (ordinarily described as red shift) as B recedes, there is a spatial expansion along the axis between $\mathrm{A}$ and $\mathrm{B}$, and so forth for each pair of points and each viewpoint.

The implication then is that the local structure requirements on space-time do not change the a priori curvature because all the $\delta$ 's change together. Therefore curvature must be due to global effects of particular theories and outside the scope of our framework. In this context we notice that the framework is relative to whatever structure of space-time exists a priori, a relative change due to acceleration associated with local masses.

Is expanding 3-space in conflict with the conservation of space-time principle? The frame of co-moving observers is not strictly homogeneous as it expands or contracts with time, so cannot be used directly. The acceleration of B with respect to $\mathrm{A}$ in one particular direction implies that in A's coordinates the space at B is distorted only along that direction, not transverse to it, and so conserved space-time may still apply as written in (10). Put another way, A could attribute recession of $\mathrm{B}$ and $\mathrm{C}$ from $\mathrm{A}$ to relativistic effects, but attribute increasing distance between $\mathrm{B}$ and $\mathrm{C}$ merely to their being farther away from A with a fixed angular separation (in a nearly flat space). But B and C would then attribute their mutual recession to relativistic effects and each of them view the other pair in ordinary recession.

An alternate analysis would be to use a composition of multiple frames of reference. Let $\mathrm{B}^{\prime}$ and $\mathrm{C}^{\prime}$ be tethered observers at a fixed proper distance from $\mathrm{A}$ in the direction of $\mathrm{B}$ and $\mathrm{C}$, respectively. A may infer transverse spatial changes about $\mathrm{C}^{\prime}$ by using observations from $\mathrm{B}^{\prime}$. But by the time these reach $\mathrm{A}$, the red shift is compounded. Figure 2 shows only a 2-D representation of 3-D space, but in actual 3-space a fourth point $\mathrm{D}^{\prime}$ would be needed to establish gravitation induced expansion in all three spatial dimensions, and it would require an additional level of composition of reference frames to establish expansion in 3 spatial dimensions concurrently. So the thus-composed time dilation would be tripled, and conservation of space-time would be satisfied.

Another point of interest is that by this method, if the acceleration remains constant at some fixed point such as B', then space there is not expanding, but statically expanded. As these questions involve global field strength, specifically excluded from our strong-field framework, we will leave further exploration of many interesting cosmological questions to future work, and the remainder of our analysis will be static. 


\subsection{Energy relations to the coefficients}

We have a relation for energy at the top and the spatial coefficient using (3) and the definition of work:

$$
\mathrm{d} E=F \mathrm{~d} l=F \rho_{r r} \mathrm{~d} r \Rightarrow \frac{\mathrm{d} E}{\mathrm{~d} r}=F \rho_{r r} .
$$

There are several ways of getting a relation to the time coefficient. The easiest one is to admit the Planck relation $E=h f$ and observe that frequency is the inverse of time, so $E=h f=h f_{r} / \varsigma=m c^{2} / \varsigma$. Taking the derivative with respect to $r$ :

$$
\frac{\mathrm{d} E}{\mathrm{~d} r}=\frac{\mathrm{d}}{\mathrm{d} r}\left[m c^{2} / \varsigma\right]=\frac{-m c^{2}}{\varsigma^{2}} \frac{\mathrm{d} \varsigma}{\mathrm{d} r} .
$$

\section{Results (exact static symmetric "Schwarzschild" solution)}

The validity of the framework will be demonstrated by solving for a static, symmetric gravitational field. To find the exact Schwarzschild coefficients we need to know how the field varies globally. As with the texts referenced above, this has to be a guess, but one which is compatible with Newtonian gravity and has an appealing physical basis. In the author's opinion, too much has been made out of "the simplest" or "the only" possible equation, but it works well enough to assume Gauss' law regarding the conservation of the force density over enclosing surfaces which gives the usual Newtonian force law in terms of the coordinate radius:

$$
F=G m M / r^{2} .
$$

Using (9) and (13) in (11) and setting it equal to (12) gives the differential equation

$$
k_{r \varsigma} \varsigma \frac{G m M}{r^{2}}=\frac{-m c^{2}}{\varsigma^{2}} \frac{\mathrm{d} \varsigma}{\mathrm{d} r} \Leftrightarrow \frac{\mathrm{d} \varsigma}{\mathrm{d} r}=\frac{-G M k_{r \varsigma}}{c^{2}} \varsigma^{3} \frac{1}{r^{2}} .
$$

Using an online differential equation solver ${ }^{1}$ we have

$$
\varsigma=\left(\frac{r}{\left(-2 G M k_{r \varsigma} / c^{2}\right)\left(1-k_{1} r\right)}\right)^{1 / 2} .
$$

To match Newton at large $r$ let $k_{r \varsigma}=1$ and integration constant $k_{1}=c^{2} / 2 G M k_{r \varsigma}$

$$
\begin{aligned}
\varsigma & =\left(\frac{r}{\left(-2 G M / c^{2}\right)\left(1-c^{2} r / 2 G M\right)} \frac{1 / r}{1 / r}\right)^{1 / 2} \\
& =\left(1-2 G M / c^{2} r\right)^{-1 / 2}=\rho_{r r}=\rho_{t t}^{-1} .
\end{aligned}
$$

Substituting (16) into (1) using the definitions (2) gives the exact Schwarzschild metric.

\section{Discussion}

\subsection{Evaluation of the method}

We have followed the spirit of the GR-Schwarzschild approach in important respects, restricting the choice of coordinates only so that conservation principles are easy to apply and easy to notice. Our coordinate relations were a crude analog of tensors suitable for simple problems. We used differential geometry but not all the flexibility it provides. Our field strength postulate is similar to the heuristic basis of many treatments. The spherically symmetric restriction yields an ordinary differential equation instead of a set of differential equations.

Finally we arrive at the exact Schwarzschild metric without any overly simple shortcuts. The differential equation (14) is sufficiently complex to introduce new order terms [9].

The use of the coordinate quantity approach has not been studied analytically to the degree tensors have, but lends itself to numerical methods and its computation of light bending has been verified to 2nd order [13].

1 wWw.symbolab.com/solver/ordinary-differential-equation-calculator/. 


\subsection{Implications of the method for theory development}

It seems odd to the author that we are told to give up the notion of gravity as a force in favor of a geometric theory, then in looking for a justification for the overall field strength to be told "The starting point must be Newton's inverse square ... expressed in differential form as Poisson's equation" ([2], p. 221, emphasis Rindler's), and finally to have to use literally the force relation (13) to solve for the critical coefficient that explains so much. Nor is this just a quirk of our minimalist method since 4 of 6 textbooks surveyed heuristically derived the field equation from Poisson's equation. In fact our method provides a quick way to evaluate the feasibility of an alternative gravity. Two examples follow.

The new framework makes it possible to postulate a field according to its effect on time (dilation), or energy which by the Planck relation is related to time, and the principle of locally conserved space-time (10) guarantees that the appropriate spatial effect will arise. How many theories have been relegated to the dust bin because they failed to progress beyond "weak fields"? However, that primarily is an advantage for "quick look" analysis. In the long run it does not alleviate the necessity of eventually finding the linkage to space and explaining the conservation law. (Since GR is not a constructive theory but a principled one it is largely pardoned from explaining itself.)

To keep things simple we begin with either a hypothetical or existing field $\Phi$ which is in some kind of stress $\Phi_{S}$ that obeys Gauss' Law $\oint_{\partial V} \Phi_{S} \mathrm{~d} A=k_{\Phi}$ and the Poisson equation $\nabla \cdot \Phi_{S}=k_{\Phi} \mu$ where $\mu$ is source mass-energy density. But for the examples we stipulate two things. First that $\Phi$ is not the space-time field. That is already satisfactorily worked out and indeed the Einstein field equation is likely the simplest variant. However, $\Phi$ can be directly related to space-time, like a coupled field, but the field equation is for $\Phi$ not space-time. Second, that the stress $\Phi_{S}$ not be force or acceleration per se, but some as yet unspecified stress in a quantum field that will give rise to such classical notions through coordinates and measurement. The intent is to demonstrate any variation other than (13) but to retain the calibration of Newtonian gravity at normal radii and expect differences only at very great field strength.

We assert that some path integral $\int_{\infty}^{r} \Phi_{S} \mathrm{~d} r$ represents the classical quantity of interest, and we will consider both energy $E$ and coordinate inertia $m_{c}$ as the development is similar but the results differ in an interesting way.

\subsection{Alternative gravity example 1: energy-field gravity (potential)}

If a field imparts some kind of energy to objects or takes it from them (e.g., potential energy), then this could produce gravitational motion (consider a Hamiltonian or Lagrangian formulation). For $E$ we take the rest energy at infinity and the usual potential energy formulation which is negative, dividing by $m$ so that it becomes an operator potential applicable to any mass:

$$
\frac{E}{m}=\frac{h f}{m} c^{2}-\frac{G M}{r} .
$$

Since time is the inverse of frequency $\mathrm{d} \tau \propto f^{-1}$ we already can form the time coefficient for the energy approach:

$$
\mathrm{d} \tau / \mathrm{d} t=\left(1-G M / c^{2} r\right) .
$$

The well-known approximation $\left(1-G M / c^{2} r\right) \approx\left(1-2 G M / c^{2} r\right)^{1 / 2}$ allows us to quickly assess that we have behavior similar to GR but with the radius of the event horizon half as large at $G M / c^{2}$. As far as the author knows, we have no observational data which could distinguish the two cases since the masses of neutron star and black hole binaries are usually not known much better than a factor of two in the limited gravitational wave data [14,15], and such objects are too far away and often too obscured for accurate observations of something like light bending [13]. We call this energy formulation "potential" gravity. However formulating the field this way leads back to force as the $\Phi_{S}$ field, which when Gauss/Poisson is applied leads somewhat more logically to GR, and so unless a different and credible $\Phi_{S}$ is found the exercise has illustrative value mainly.

\subsection{Alternative gravity example 2: relative-inertia-field gravity (proximity)}

Inertia fields, on the other hand, are the subject of intense theoretical development in at least two directions. The Higgs field technically has no deep connection to gravity as it only reapportions energy as rest mass [16,17]. But the vacuum-reaction hypothesis [18] has been recognized by its authors as gravity related, and developed as a typical weak-field gravity [19], labeled "pedagogical" probably because it lacks the spatial component which our framework can provide.

For this example we formulate inertia not as proper rest mass, but as our coordinate inertia $m_{c}$, the effective resistance to motion as inferred by remote observers in their coordinates. Since this resistance increases with time dilation, instead of negative potential as in (17) we have increasing coordinate inertia. We add the potential and divide by $c^{2}$ to obtain inertia-mass units, giving the relative coordinate inertia:

$$
\frac{m_{c}}{m}=\frac{m}{m}+\frac{G M}{r c^{2}}=\left(1+G M / r c^{2}\right) .
$$


Note that the coordinate inertia $m_{c}$ is not to be confused with energy, and cannot be used to compose the energy of a system as the sum of its parts. That would have been appropriate with the potential method above. The coordinate inertia is only an indication of resistance to motion which some remote observer may see, and only for motion relative to the mass $M$.

With $\mathrm{d} \tau \propto m_{c}$ the metric time ratio has the above factor (19) in the denominator:

$$
\mathrm{d} \tau / \mathrm{d} t=\left(1+G M / c^{2} r\right)^{-1}
$$

A similar approximation holds, and while the event horizon radius is zero, it is still likely the above comments about observations to date hold since direct observations do not come from beyond or even at the horizon, but from in-falling matter. The unique "additive" nature of this variant makes a known approximation of Mach's Principle into an exact one [20]. We therefore will call this variant "proximity" gravity, and note that the yet-to-be-discovered $\Phi_{S}$ spin-2 field would not be force as the sign is wrong. From a philosophical view, this notion is more consistent with gravity not being a force. The Poisson/Gauss conserved $\Phi_{S}$ tension would be a stress that causes the spatial changes, perhaps stochastically via the decay of spin alignment (coherence) in expanding volumetric shells, giving the correct Newtonian limit. Some novel spin architectures other than simply a new spin-2 boson have been suggested but not developed [10].

\section{Verification}

There are two separate issues. The first and main issue is distinguishing among members of the family, which could in principle be arbitrarily close. We will parameterize this below. The Parameterized Post-Newtonian Approximation $(\mathrm{PPN})[21]$ is the gold standard for comparing and verifying metric gravity theories and is general enough to handle any of the cases we have mentioned. However it is quite complex and most of the work to date has used it for astrophysical and cosmological problems that do not involve the strong-field situations which constitute the differences in members of our framework. Here we will propose a single-parameter criterion to distinguish members of our minimalist strong-field framework.

The second issue is verification of the "minimalist" framework itself.

The approach guarantees that in weak fields family members behave in the same manner as GR because the weakfield approximations are identical. Experimental data validated to high order in $v / c$ using linearized GR [22] as in the case for gravitational wave detections, is still limited by the weak-field character of its linearized GR basis. Also note that at present the source masses and initial orbits have large uncertainties [23,24], and as we will see in this section, one family member might mimic another quite well if the source masses are are not well known. We will attempt to quantify those effects. There has to date been no dynamical study (that the author can find) which compares, for example, a GR black hole absorption and ring-down with one of the alternatives in which the object masses are slightly different in a way that might produce a similar ring-down characteristic from a different strong field. Limits on PPN coefficients are usually not given in experimental papers. Here we try to give some verification rules-of-thumb consistent with our "quick-look" evaluation philosophy.

The discussion here addresses only strong fields. There are important verification issues in GR which are still outstanding but not addressed as strong-field issues, for example the Lens-Thirring effect. This has been measured near Earth to an accuracy of 19\% [25-27]. However, the magnitude is much less than other factors in attempts to measure 2 nd order effects $[14,15]$ and so we do not consider it. Lense-Thirring is based on a linearization and analogy to electromagnetism which is true for any metric theory [26], and so the weak-field effects would be expected to be indistinguishable for members of the framework.

\subsection{Single-parameter approximation for framework members}

The two alternatives plus Schwarzschild just happen to fit in a framework based on the single parameter $n$, defined as follows (and illustrated as plots of $\varsigma$ in fig. 3 ):

$$
\begin{aligned}
\varsigma & =\left(1+n G M / r c^{2}\right)^{1 / n} \\
n & =-2 \Rightarrow \text { Schwarzschild-GR } \\
n & =-1 \Rightarrow \text { potential-gravity } \\
n & =1 \Rightarrow \text { proximity-gravity. }
\end{aligned}
$$




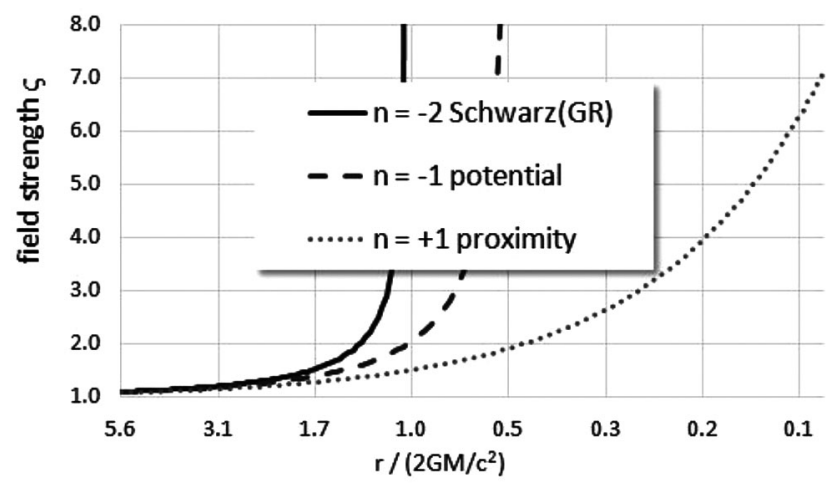

Fig. 3. Field strength comparison of framework members.

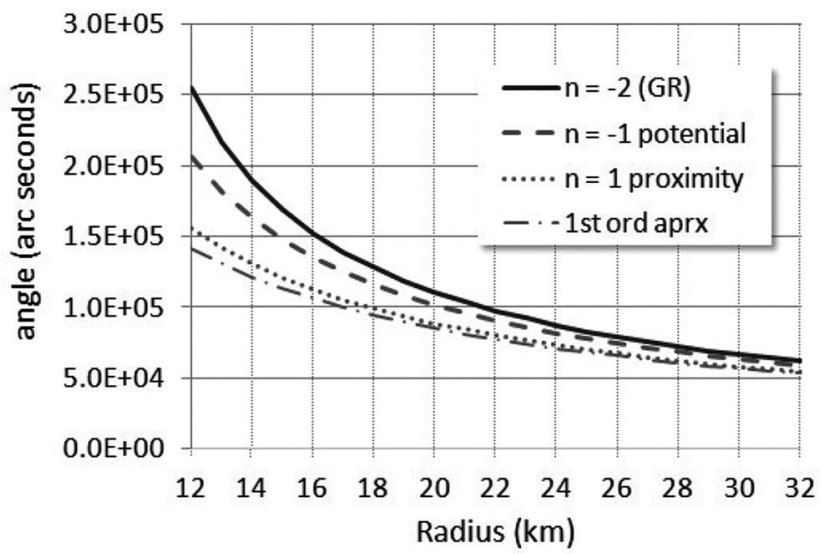

Fig. 4. Light bending near a 1.4 solar mass neutron star for three framework members.

\subsection{Optical test possibilities}

Figure 4 gives a plot of light bending angles for a 1.4 solar mass neutron star with a 12 kilometer radius, plotted for nearest approach radii of 12 to $32 \mathrm{~km}$, for $n=-2, n=-1, n=1$ done with a numerical method [13], compared with the analytic 1st-order GR approximation.

It is apparent that the $n=1$ case bending is just slightly more than 1st-order GR, while the full (all-order) GR bending is about $2 / 3$ greater within a kilometer or two of the star. While it would be difficult to ascertain all parameters in such a case, it appears that the shape of the bending curve as a function of the closest approach of the light ray might be used as a discriminator even in the presence of a good bit of measurement uncertainty. Resolution of a few kilometers at exosolar distances is not currently possible. Even using a space mission to $550 \mathrm{AU}$ to take advantage of the sun as a gravitational lens, resolution of a 12 meter dish of the order of a millionth of an arc second [28] amounts to 2 kilometers at a light year, and most neutron stars are hundreds of light years distant, implying interferometry techniques over a kilometer or more would be required. With the capability of interferometry approaching the microarcsecond range [29], the required resolution might be obtained to a range beyond 1000 light years.

The more immediate strong-field data will be gravitational wave data, which has the difficulties previously discussed. Disambiguation of source mass and field strength may be possible for specific theories, or under the assumption that framework members behave dynamically like GR, but that is piling on a lot of assumptions.

\subsection{Precession tests and dark matter distributions}

It has recently been suggested that precession in orbits of stars as close as 140 astronomical units (AU) to the supermassive black hole at the Galactic Center may be useful as a strong-field test [30]. The mass is thought to be 4 million solar masses. But still the field strength is about 2.5 times weaker than in the neutron star example used for light bending, implying that the shape of data curves will not be able to distinguish theories and overcome various data uncertainties. Figure 5 shows relative precession comparisons (neglecting spin) as percent deviation from GR. 


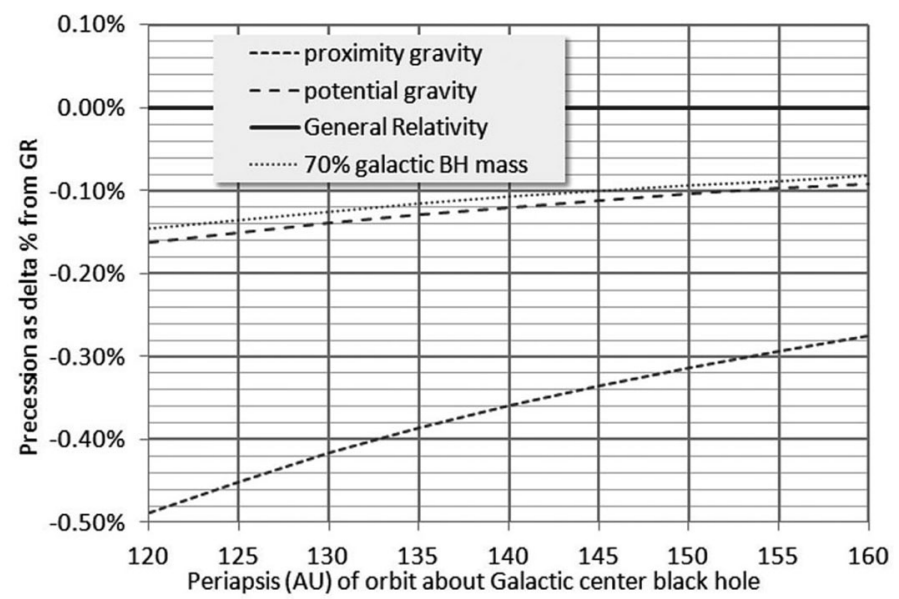

Fig. 5. Relative precession for three framework members near Galactic Center.

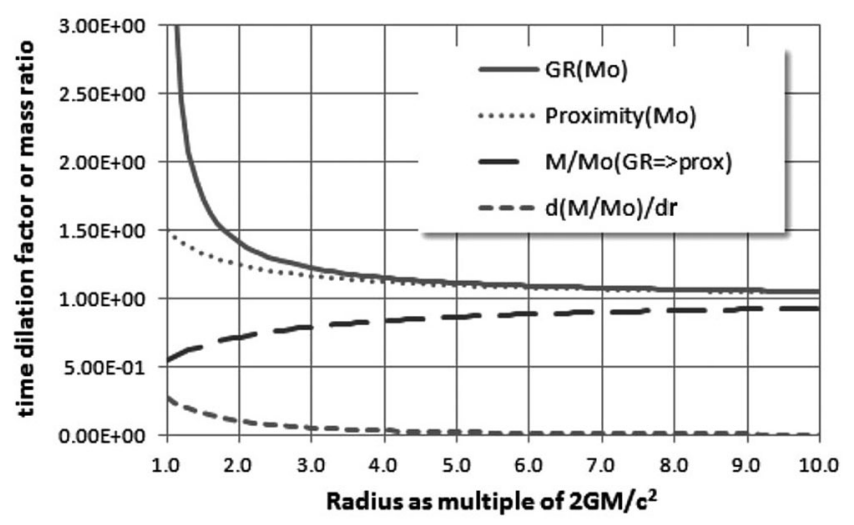

Fig. 6. Mass distribution required for GR to mimic proximity gravity.

There is only a 0.15 to 0.5 percent difference for framework members at a periapsis corresponding to core star S0-2. That the curve shapes are not an adequate discriminator is illustrated by including a plot for GR precession with only $70 \%$ of the expected central mass (only 15 years ago that was approximately the mass estimate in use), which almost exactly overlays the potential gravity framework member.

The issue of dark matter distribution around the core has been raised [31] limiting the accuracy of tests below significance thresholds at this time [25,32-35]. If a significant fraction of the dark matter were within the orbit, this would affect the result. Any framework member may mimic a weaker framework member if a specific distribution of mass exists outside the black hole. In fig. 6 we calculate the mass distribution for GR to mimic proximity gravity. The total central mass is $M_{0}$, but the collapsed amount is half that. The parameter $M$ is the amount of mass (collapsed plus distributed) enclosed at radius $r$.

The expected GR field strength based on the total mass being collapsed, the upper solid line, would not be seen. Instead, the dotted line would be seen. We have a choice of explanations, a different strength of gravity, a dark matter distribution, or perhaps some new force. This situation could be differentiated if the mass distribution could be determined using only weak-field gravity (which does not seem likely) or another method (not likely in the case of noninteractive dark matter), or if the actual location of the event horizon could be inferred, perhaps from measurements inside what would have been the horizon of an object of mass $M$, though even this does not pin down all ambiguity.

\subsection{Is the locally conserved space-time principle verifiable?}

The simplest though certainly not the only way to proceed is to split our 1-factor framework approximation into separate temporal $(n)$ and spatial $(w)$ parameters:

$$
\begin{aligned}
\varsigma & =\rho_{t t}^{-1}=\left(1+n G M / r c^{2}\right)^{1 / n}, \\
\rho_{r r} & =k_{r \varsigma}\left(1+w G M / r c^{2}\right)^{1 / w} .
\end{aligned}
$$


The $k_{r \varsigma}$ is the part which is a function of coordinates, a constant in the case of Schwarzschild, and the locally conserved space-time principle (10) is true if and only if $k_{r \varsigma}=\rho_{r r} \rho_{t t}=\rho_{r r} \varsigma^{-1} \Leftrightarrow n=w$.

Notice that a great deal of testing of the conservative space-time principle has been done, starting with Eddington. If light had not been "double bent" by gravity that would correspond to $w \rightarrow \infty$ taking the spatial factor out of play. Most weak-field gravities that have only time dilation can be described as $w \rightarrow \infty$. Solar light bending is 1750.4 milliarcseconds with an experimental uncertainty of 0.03 milliarcseconds [36]. The second-order component of solar light bending is approximately 0.006 milliarcseconds [13], or $1 / 5$ th as large as the current experimental capability. The European Space Agency GAIA mission launched in 2013 has the potential, by measuring billions of stars to 20th magnitude, to reduce the error to the order of 0.001 milliarcseconds, which would seem adequate, but there are still two problems. First, if instead of $w \rightarrow \infty$ we have some value closer to $n$, then the problem is harder. Second, using optical means, distinguishing two framework members $n=1$ and $n=-2$ is essentially the same problem as distinguishing $w=1$ and $n=-2$. So that means we have to distinguish in a given set of data whether we have detected a conservative space-time principle violation, or merely that gravity is a different member of the framework than expected. This could be determined by an independent measure of field strength, such as time dilation, if sufficiently accurate data is available.

\section{Conclusion}

The first result of this paper's approach is that the local structure of space-time is found to be a fundamental consequence of conservation principles, and not merely a coordinate artifact. This result is limited to space-time regions not hidden behind coordinate singularities, which is not a serious limitations as such singularities prevent experiments of most kinds, including those concerned with conservation principles. Should a wormhole be demonstrated traversable by energy or information without a singularity boundary, and without the "no-communication" limitation of entanglement, then obviously this result would need revisiting. The two postulates leading to this result are the conservation of proper momentum and consistency of field strength postulates.

The second result of the approach is that static symmetric solutions for global field postulates are relatively easy to work out, without needing a full development. This is demonstrated by finding a global field strength postulate corresponding to GR and finding the exact Schwarzschild solution from it, using only one differential equation along the way. It is apparent the other components of the usual Schwarzschild method in GR are necessary to work out the local space-time structure. The flexibility of the traditional development, while providing advantages in practical situations, obscures what results are coordinate artifacts and what are genuine results. Further examples of two other field strength postulates are provided which are even simpler, one of which, potential gravity, is mainly a historical pedagogical example, and the other of which, gravity from inertia, illustrates a variation of theoretical interest. Although, as it differs from GR by almost the greatest amount allowed in our "family" of metric gravities, it may be the first to fall as more accurate measurements of strong fields become available.

And the third result of the approach is exactly that, theoretically interesting verification targets of varying degrees of closeness to GR, which by conception already meet all weak-field empirical data already obtained.

Fourthly the conservation of proper momentum and consistency of field strength postulates can simplify the process of developing and evaluating complete metric or metric-like field theories because the spatial metric arises from the temporal one through conservation principles. This avoids the problem of various field gravities only being applicable to weak fields which some alternative developments encounter.

The fact that in potential and inertia gravity a static solution may be found without solving a differential equation suggests that there may be a class of theories that can have fewer internal dependencies than GR, which is so far unstable in quantum formulations, which might have implications for quantum field theories of gravity. This is one area for possible future investigation. A systematic way of deriving full tensor field equations and wave equations corresponding to a particular field strength postulate is another.

The author thanks Eric Lord for review and comment on the final paper, Klaus Kassner for a tether analysis of force in GR, John Macken for draft reviews and discussions on the coordinate velocity of light, Clifford Will for email discussions on the role of equivalence, Richard Price for comments on a very early draft, Gerald Cleaver for review of a precursor paper, and for general encouragement on the subject matter: Charles Francis, Stefano Quattrini, Jan Cerzniawski, W.T. Elam, Luca Bombelli, Matts Roos, Robert J. Low, Max K. Wallis, Douglas L. Weller, Allen D. Allen and Clifford Chafin. The author also thanks an anonymous reviewer for additional references and numerous helpful suggestions.

\section{Conflict of interest}

The author declares there are no conflicts of interest. The work does not necessarily represent the opinions of the National Aeronautics and Space Administration. 
Open Access This is an open access article distributed under the terms of the Creative Commons Attribution License (http://creativecommons.org/licenses/by/4.0), which permits unrestricted use, distribution, and reproduction in any medium, provided the original work is properly cited.

\section{References}

1. L.D. Landau, E.M. Lifshitz, The Classical Theory of Fields, 4th ed., Course of Theoretical Physics, Vol. 2, translated by M. Hamermesh (Butterworth-Heinenann Reed Intl., Oxford, 1975).

2. W. Rindler, Relativity Special, General, and Cosmological, 2nd ed. (Oxford University Press, Oxford, New York, 2006).

3. R. D'Inverno, Introducing Einstein's Relativity (Oxford University Press, NY, 1998).

4. S. Weinberg, Gravitation and Cosmology: Principles and Applications of the General Theory of Relativity (Wiley and Sons, New York, 1972).

5. M. Blau, Lecture Notes on General Relativity (Institute for Theoretical Physics, University of Bern, Switzerland, 2014) http://www.blau.itp. unibe.ch/Lecturenotes.html.

6. C.W. Misner, K.S. Thorne, J.A. Wheeler, Gravitation (W.H. Freeman \& Co., San Francisco, 1973).

7. L. Iorio, Universe 1, 38 (2015).

8. I. Debono, G.F. Smoot, Universe 2, 23 (2016).

9. R.P. Gruber, R.H. Price, S.M. Matthews, W.R. Cordwell, L.F. Wagner, Am. J. Phys. 56, 265 (1988).

10. R.L. Shuler, Theor. Phys. 2, 51 (2017).

11. J. Hanc, S. Tuleja, M. Hancova, Am. J. Phys. 72, 428 (2004).

12. R.L. Shuler, Adv. Astrophys. 1, 47 (2016).

13. R.L. Shuler, Adv. Astrophys. 2, 76 (2016).

14. B.P. Abbott et al., Phys. Rev. Lett. 116, 061102 (2016).

15. P. Astone et al., Gen. Relativ. Gravit. 47, 11 (2015).

16. P.W. Higgs, Phys. Lett. 12, 132 (1964).

17. S. Weinberg, Phys. Rev. Lett. 19, 1264 (1967).

18. A. Rueda, B. Haisch, Phys. Lett. A 240, 115 (1998).

19. H.E. Puthoff, Found. Phys. 32, 927 (2002).

20. R.L. Shuler, Phys. Educ. 50, 88 (2015).

21. C.M. Will, Pramana-J. Phys. 63, 731 (2004).

22. C.W.F. Everitt et al., Phys. Rev. Lett. 106, 221101 (2011).

23. L. Iorio, EPL 96, 30001 (2011).

24. G. Renzetti, Open Phys. 11, 531 (2013).

25. L. Iorio, Galaxies 1, 6 (2013).

26. O. Chashchina, L. Iorio, Z. Silagadze, Acta Phys. Pol. Ser. B B40, 2363 (2009).

27. J.H. Taylor, L.A. Fowler, P.M. McCulloch, Nature 277, 437 (1979).

28. C. Maccone, J. Brit. Interplanet. Soc. 47, 45 (1994).

29. M. Kopeikin, V.V. Makarov, Phys. Rev. D 75, 062002 (2007).

30. A. Hees et al., Phys. Rev. Lett. 118, 211101 (2017).

31. V.I. Dokuchaev, Y.N. Eroshenko, K.S. Klimkov, Phys. Proc. 74, 292 (2015).

32. L. Iorio, Mon. Not. R. Astron. Soc. 472, 2249 (2017).

33. F. Ferrer, A. Medeiros da Rosa, C.M. Will, Phys. Rev. D 96, 083014 (2017).

34. L. Sadeghian, F. Ferrer, C.M. Will, Phys. Rev. D 88, 063522 (2013).

35. T. Lacroix, Dynamical constraints on a dark matter density spike at the Galactic Centre from stellar orbits, arXiv:1801.01308 (2018).

36. C.M. Will, Class Quantum Grav. 32, 124001 (2015). 